\title{
FUNNet - A Novel Biologically-Inspired Routing Algorithm Based on Fungi
}

\author{
More efficient network management solutions
}

\author{
Xu Hao, Ruth Falconer, David Bradley \\ School of Computing \& Creative Technologies \\ University of Abertay Dundee \\ Dundee, UK \\ h.xu@abertay.ac.uk,r.falconer@abertay.ac.uk, \\ d.bradley@abertay.ac.uk
}

\begin{abstract}
Future data communication networks show three emerging trends: increasing size of networks, increasing traffic volumes and dynamic network topologies. Efficient network management solutions are required that are scalable, can cope with large, and increasing, traffic volumes and provide decentralised and adaptive routing strategies that cope with the dynamics of the network topology. Routing strategies are an important aspect of network management as they have a significant influence on the overall network performance. This paper introduces the preliminary studies for FUNNet, a new routing algorithm inspired by the kingdom of Fungi. Fungi form robust, resilient and responsive networks and these networks change topology as a consequence of changes in local conditions. Fungi are capable of expanding in size as they self-regulate and optimise the balance between exploration and exploitation which is dependent on the transport of the internal resource, i.e. 'traffic', within the network. FUNNet exploits the biological processes that are responsible for simulating fungal networks in a bio-inspired routing protocol. The initial results are positive and suggest that fungal metaphors can improve network management, although further evaluation of more complex scenarios is required.
\end{abstract}

Keywords-biologically-inspired, network, routing, fungi

\section{COMMUNICATION NETWORKS AND CHALLENGES IN TECHNOLOGY}

Data communication networks are developing rapidly and this generates a range of new challenges in relation to network management and routing. These developments are associated with three major trends; the increasing size of networks, their associated increase in traffic volumes, and dynamic network topologies.

Wide area networks, such as the Internet and cellular mobile networks, are increasing in size and the volume of traffic, therefore increasing the complexity of the network topology. One estimate of the expected growth in internet users for the period 1995 to 2010 is shown in Fig. 1 [1].

Users are also demanding a wider and wider range of services from networks. This is especially so in relation to the requirement to be able to access multimedia content, which generates further pressures

\author{
John Crawford \\ Faculty of Agriculture, Food and Natural Resources \\ University of Sydney \\ Sydney, Australia \\ j.crawford@usyd.edu.au
}

on network traffic and its management. Fig. 2 [2] gives an estimate of the anticipated growth in internet services up to 2010, including the growth of new services such as internet video to television.

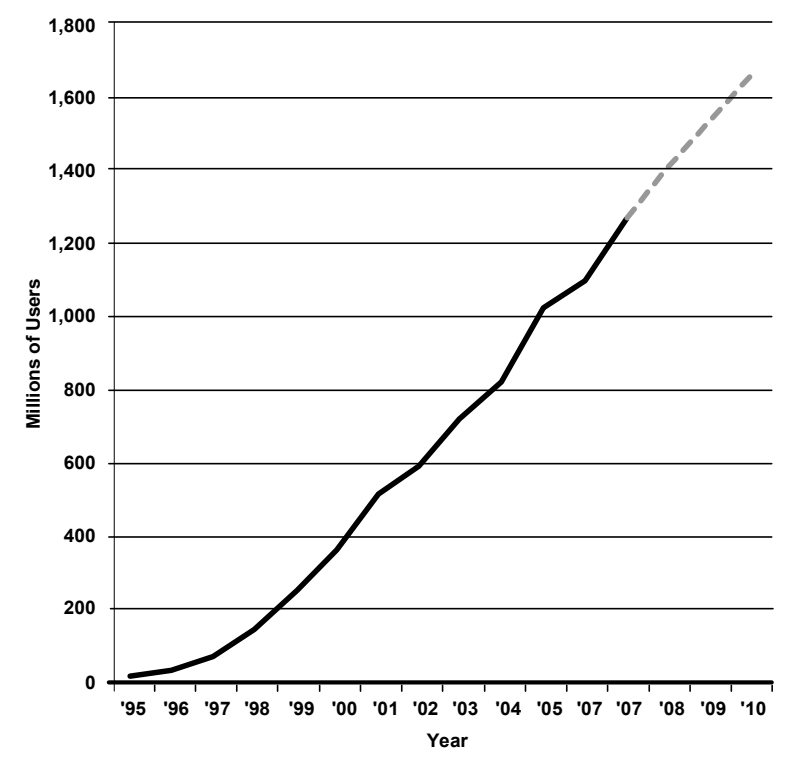

Figure 1. Growth of Internet users [1]

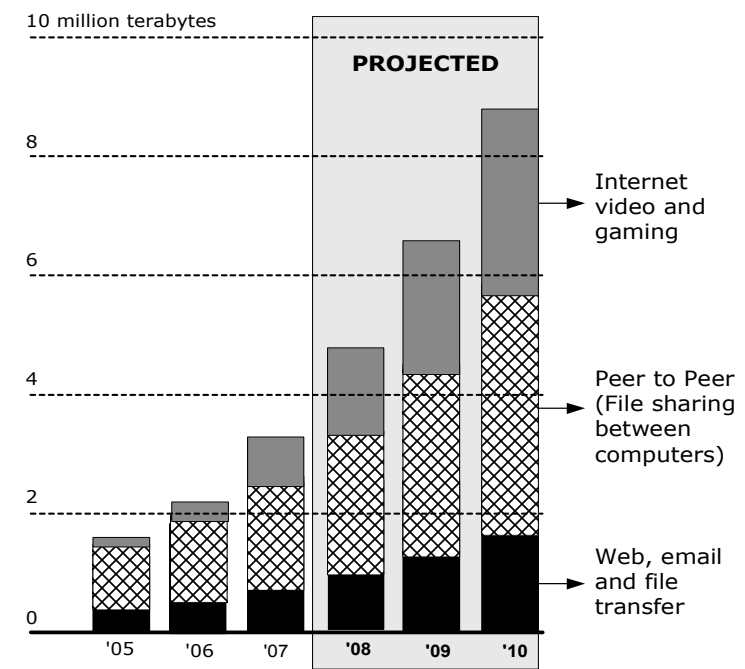

Figure 2. Predicted growth of Internet traffic [2] 
Finally, the increasing adoption of mobile facilities requires a network that can dynamically adapt its topology. A mobile node can join or drop out of a network, based on its position resulting in a network with frequent changes in topology. The network must be flexible enough to adapt to these changes within an appropriate time frame.

Further, these trends in the development of communication networks are not independent, and a network will not only expand its size, but also provide a multiple range of services for users while adapting its connectivity.

These challenges for communication networks demand new tools and techniques for network management. One important aspect of network management is the routing algorithms that organise the traffic in a network efficiently. New routing algorithms are required to meet the changing environment.

This paper has 7 sections. Section II provides a brief review of biological inspired solutions for communication networks. Section III introduced fungal networks, and suggests possible inspirations from fungal network. Section IV introduces FUNNet, a new routing algorithm inspired by fungi. Section $\mathrm{V}$ tests the FUNNet by apply this routing algorithm into a 30 node network model. The results of this test are compared with ANTNet [3] demonstrated in Section VI. Section VII suggests further work of this project.

\section{BIOLOGICAL INSPIRED SOLUTIONS}

Routing algorithms such as ANTNet [3], inspired by biological systems, have been generated to search for an optimised routing solution. Biologists observed that biological systems have the ability to self-organise, which could generate patterns at the higher levels of a system merely from the numerous interactions among the lower-level components of the system. In this context, Camazine et al suggest that:

"Moreover, the rules specifying interactions among the system's components are executed using only local information, without reference to the global pattern" [4].

For example, an ant colony can build a nest with a very complex structure that suggests a level of management beyond the capacity of any individual ant and its 'intelligent' ability to 'design'. But through simple pheromone based communications between individual ants, the colony builds a fully functional nest.

What if the ability to self-organise of a biological system could be adapted into a network system? Is it possible to improve system performance? Much research effort has been driven by this curiosity [1][5][6] and ANTNet is one of the most well known and successful examples of a biologically inspired solution.
As the aim at least in the initial stage, was to look at the potential for biologically driven algorithms, ANTNet was chosen as the basis for the first comparative of these studies, the scope of the comparison would then, at some future date be expanded to encompass other, more complicated routing algorithms.

\section{Potential SOLUTIONS}

Not only swarm societies such as ants have the 'intelligence' mentioned above, other biological systems such as fungi also demonstrate self-organized and complex network structure. Barr classified fungi as one of the six kingdoms of life [7], and they are one of the most successful groups of organisms on earth [8].

The success of fungi is largely related to the form of fungi growth. Fungi typically grow as filaments, termed hyphae (singular: hypha), which extend only at their extreme tips [9]. Fungal hyphae grow and branch, and the branches in turn branch, to form a network of hyphae known as the mycelium [10]. The fungal mycelium is a combination of two types of biomass: immobile biomass and mobile biomass. Immobile biomass is the material that is used to construct the hyphal tube, through which the mobile biomass then flows.

Such a fungal network (mycelium) is able to keep extending as long as its hyphae are growing and branching. A fungal colony has been found in Canada which extends to an area of 2 square kilometres.

A fungal network is a dynamic network, varying its location, size and structure according to environmental conditions. Internal mobile biomass traffic is kept in balance and in turn reshapes the structure of fungal network. In other words, a fungal network can adapt its structure in response to environmental changes by controlling the mobile biomass traffic inside. For example, a fungal network can grow across areas with low nutritional status by routing its internal resources to the growing tips to build a 'bridge' to that area.

These processes emerge, apparently without any central control, and can potentially be used to inspire communication network design, operation and maintenance.

The original mathematical model encapsulating growth and development of fungal colonies developed by the group, Falconer et al 2005 [11], was further developed, to provide a hyphal-level description of the fungal network. The hyphal-level model reproduces many of the observed behaviours, i.e. concentric peaks of biomass as shown in Fig. 3 [10]. This discrete hyphal-level formulation is suggestive of a mapping between fungal and communication networks set out in Table 1 which summarizes the general ideas those of how a fungal network would represents a communication network. An initial test of selected mappings has been done through FUNNet. The selected 
mappings covered key aspects of network routing whilst maintaining simplicity.

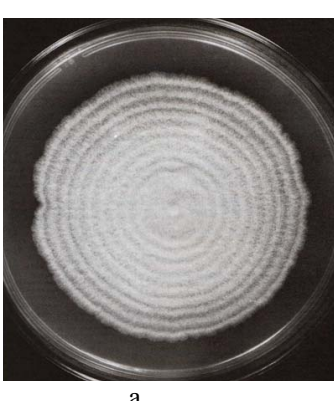

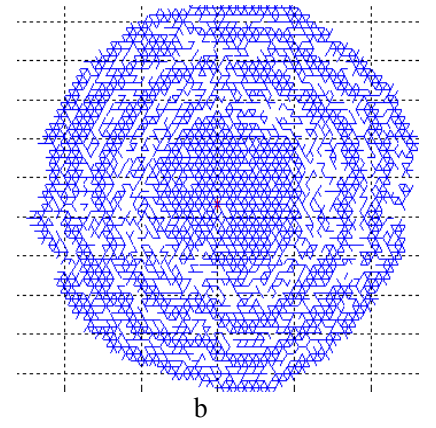

Figure 3. Ring patterns for fungal growth a. real fungus [10]; $b$. hyphal level simulation

TABLE 1. POTENTIAL RELATIONSHIPS BETWEEN FUNGAL AND COMMUNICATION NETWORK

\begin{tabular}{|c|c|}
\hline Fungal network & Communication network \\
\hline Hyphae & $\begin{array}{l}\text { - } \text { Connection between nodes } \\
\text { or } \\
\text { - Local network information held by } \\
\quad \text { nodes }\end{array}$ \\
\hline Hyphal tips & $\begin{array}{ll}\text { - } & \text { Nodes that are loading and } \\
\text { sending data } \\
\text { - } \quad \text { Network information at terminals }\end{array}$ \\
\hline Mycelia & $\begin{array}{ll}\text { - } & \text { Entire network } \\
\text { or } & \\
\text { - } & \text { Routing table } \\
\end{array}$ \\
\hline Mobile biomass & $\begin{array}{l}\text { - Data traffic trail } \\
\text { and } \\
\text { - Have a potential to transfer into } \\
\text { immobile biomass }\end{array}$ \\
\hline $\begin{array}{l}\text { Mobile biomass } \\
\text { transport } \\
\text { mechanisms }\end{array}$ & Rules for data routing \\
\hline Immobile biomass & $\begin{array}{l}\text { Traffic capacity or node's ability to } \\
\text { attract data flow }\end{array}$ \\
\hline Biomass recycling & $\begin{array}{l}\text { Interaction between the data traffic and } \\
\text { the attraction of data flow, could be } \\
\text { considered in both positive feedback or } \\
\text { negative feedback, intended to find a } \\
\text { learning pattern for network routing }\end{array}$ \\
\hline $\begin{array}{l}\text { Biomass } \\
\text { consumption }\end{array}$ & $\begin{array}{l}\text { Reducing attraction of data traffic } \\
\text { through time }\end{array}$ \\
\hline Uptake & Loading data packet \\
\hline Hyphae death & $\begin{array}{l}\text { - } \quad \text { Disconnection of links } \\
\text { - } \quad \text { Invalid of local network information }\end{array}$ \\
\hline
\end{tabular}

\section{FUNNET}

Unlike other routing algorithms, FUNNet does not have a routing table for each node containing routing information for all other nodes in the network. Each node in a fungal network only contains information relating to its next-nearest neighbours. The attributes of each node are connectivity, capacity and traffic. This is particularly significant for networks with a dynamic topology. If any node is added to or deleted from a network, it is not necessary for all other nodes to modify their routing table.

\section{A. Fungal metaphors}

Based on the mapping and interpretation of the potential links between fungal networks and communication networks set out in section 3, several fungal metaphors have been applied to FUNNet.

- The fungal mycelium represents the topology of the communication network.

- Each node has the associated parameters of mobile biomass $(\mathrm{mb})$ and immobile biomass $(\mathrm{imb})$, in which mobile biomass is supposed to be diffused in the trail of a successful routing, and immobile biomass represents the weight to attract new traffic of a node

- When a demand to send a data packet is issued in a communication network, an amount of mobile biomass will be generated. The associated data traffic will be released following arrival at its destination, but the related mobile biomass will not decay, but diffuse evenly along the route over which the data packet passed.

- The direction of a data packet transferred in a communication network is associated with the mobile biomass transport mechanisms in a fungal network.

- Data traffic always aims to flow from a region of higher mobile biomass density to one of lower density.

- Biomass recycling in a fungal network means the transformation between mobile biomass and immobile biomass. This concept is used in communication network in the way to increase local immobile biomass concentrations when the local data traffic is higher than a certain threshold, but will not decrease it even if the local traffic is lower than the threshold.

- In a fungal network, biomass consumption will decrease the immobile biomass concentration (imb) over time.

\section{B. Route selection}

In order for the data to be routed through the network from origin to destination, a routing decision must be taken each time data arrives at a node to identify the next node to pass the data to.

We propose that mobile biomass transport mechanisms can be used to describe data traffic flow. We describe the methodology in qualitative terms here, by analogy with the associated biological processes represented in our model. The governing equations are described in Section 4.4.

Firstly, in our model of the fungal mycelium, mobile biomass is passed between nodes through the 
network of hyphae. Any individual hypha is parameterised only in terms of the neighbours that directly connect to it. The local mobile biomass flows from a node according to a diffusive process at a rate determined by its neighbours' mobile and immobile biomass concentrations. In the case of the communication network model, it is also assumed that each node has knowledge of its immediate neighbours, defined in this instance as all those nodes capable of receiving a package of data from the transmitting node.

Secondly, again referring to the fungal network, the level of the immobile biomass influences the nature of the flow of mobile biomass. In the communications network, we use local immobile biomass concentration to weight the node's ability to attract data flow, and a parameter 'imb', corresponding to the immobile biomass in the fungal network, has been added to the node's parameter set to represent this.

Finally, the possibility of a particular neighbour being chosen as the next destination for the data is also influenced by that neighbour's capacity and its current traffic levels. Specifically, the higher the available capacity at the node, the higher the probability of it being chosen as the next destination node.

Combining these three elements, imb, capacity and traffic, the probability of neighbour ' $n$ ' being chosen is represented by the value of $\mathrm{P}_{\mathrm{n}}$ in equation 1

\section{Mobile biomass diffusion}

A constant amount $\mathrm{E}$, of mobile biomass will be generated when a data packet is required to be sent to its destination. When the data packet arrives at its destination, the mobile biomass will then diffuse evenly onto the nodes the packet passed through. Therefore, these nodes will increase their value of $\mathrm{mb}$ by $\mathrm{E} / \mathrm{N}$, in which $\mathrm{N}$ is the number of nodes the route passed. The changes in mb will have a further influence on the value of immobile biomass $(\mathrm{imb})$ through the process of biomass recycling.

\section{Governing equations}

Biomass recycling in a fungal network is interpreted in terms of the process used to modify the routing information in a communication network. According to equation 1 , the routing decision is dependent on a node's current traffic capacity, its current traffic as represented by mobile biomass, and the level of immobile biomass (imb). Biomass recycling could therefore represents the changing of level of immobile biomass, and hence the value of the imb parameter, influenced by current traffic. In this model, biomass recycling happens after every successful routing, and will transfer an amount ' $\lambda$ ' of mobile biomass into immobile biomass. This is represented by equation 2 .

$$
\mathrm{P}_{\mathrm{n}}=\frac{\left(\text { capacity }_{n}+i m b_{n}-\text { traffic }_{n}\right)}{\sum(\text { capacity }+i m b-\text { traffic })} \times 100 \%
$$

$$
\lambda=\alpha\left(\frac{m b+\text { traffic }}{i m b}\right)^{\theta}-\beta\left(\frac{m b+\text { traffic }}{i m b s}\right)
$$

Equation 2 implements the criteria for biomass recycling in the fungal model where $\alpha, \beta$ and $\theta$ are constants. In equation 2 , data traffic is looked upon as newly generated mobile biomass.

\section{E. Biomass consumption}

Biomass consumption reduces the value of imb in every time step when the value of imb larger than a threshold. This can be represented by equation 3 .

$$
i m b_{t}=i m b_{t-1}-C
$$

where $\mathrm{C}$ is a constant.

\section{F. Learning pattern}

Biomass diffusion, recycling, and consumption change the value of imb at each node in the network, which will further influence routing decisions. These three processes together constitute a learning pattern which is able to refine the routing decisions by generating the shortest paths. Results are judged by the average number of hops for each successful routing.

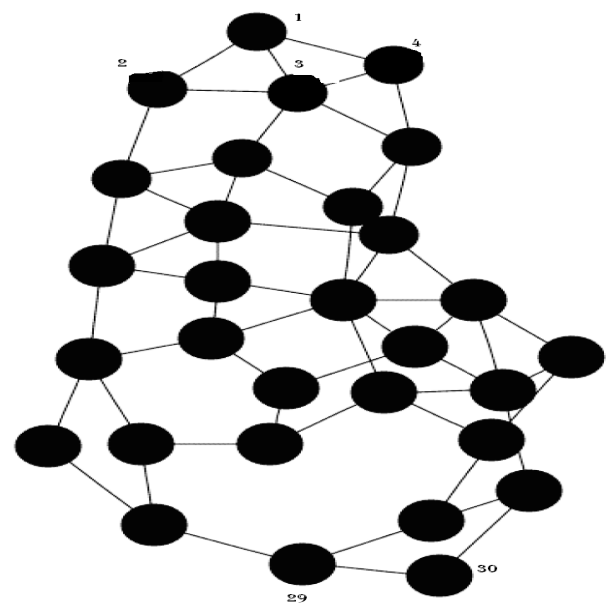

Figure 4. Topology of the network model

\section{NETWORK MODEL}

A simple communication network model containing 30 nodes has been built to test the effectiveness of FUNNet. This network is closely resembles the British Synchronous Digital Hierarchy (SDH) network [12]. Fig. 4 shows the topology of this network model. It is also consistent with what might be expected from a self-organizing sensor network based on the use of technologies such as WiFi.

At regular time intervals, a call is generated to send a data packet of the same size from a randomly selected source node to a randomly selected destination node. 
TABLE 2. COMPARISON OF FUNNET AND ANTNET

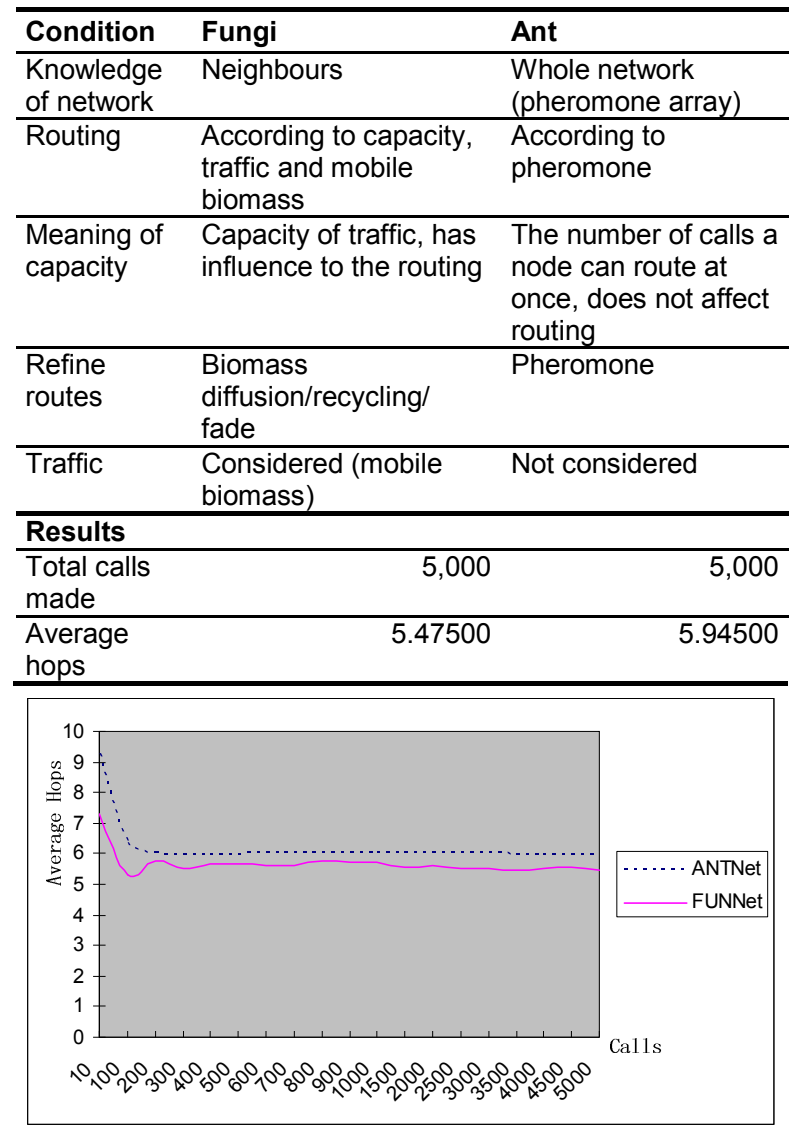

Figure 5. Comparison between ANTNet and FUNNet

\section{RESULTS}

As this is the first step towards applying the fungal metaphor to communications networks, the quality of routing is simply judged in the first instance by the average hops of a data packet in reaching its destination, and is compared with ANTNet. Other criteria to be investigated include:

- Total transaction delay

- Search time and response to network change

The results obtained depended on the parameter values and the best result which is indicated by the lowest average number of hops is of interest here. After an exhaustive search, the values of parameters that generate the best result are:

$$
\alpha=0.5, \beta=0.5, \theta=5, E=10, C=0.015
$$

Table 2 provides a comparison between the levels achieved by FUNNet and ANTNet for this simple network model. Fig. 5 demonstrates the comparable results according to the steps of number of total calls made.
This result as indicated in Fig. 5 shows the fungal metaphor has the lowest average number of hops compared with the ant metaphor. This suggests that there is considerable potential for applying fungal metaphors to improving communication network management though further evidence is needed.

\section{FURTHER WORK}

Though the work to date has demonstrated potential, further evidence is needed to prove the application of fungal metaphors in network management. As fungal metaphors are especially expected to be applicable to dynamic networks, experiments within dynamic network environments in particular are needed.

These include adding or deleting nodes from the original network, and changing traffic densities in the network. If these experiments further suggest a potential application for fungal metaphors, a wider range of topics should then be involved.

\section{ACKNOWLEDGEMENTS}

The authors would like to thank the University of Abertay Dundee and the Norman Fraser Trust for their support in carrying out the work reported in the paper. One author, Xu Hao, would also like thank his parents who always support him.

\section{REFERENCES}

[1] Internet world stats. 2008. Internet growth statistics. [online]. Available from:

http://www.internetworldstats.com/emarketing.htm [Accessed 17 Nov 2008]

[2] Cisco. 2008. Global consumer internet traffic. [online]. Available from:

http:/graphics8.nytimes.com/images/2008/03/13/technology/int ernettraffic.L.jpg [Accessed 18 Nov 2008].

[3] G. Di Caro and M. Dorigo, "AntNet: A mobile agents approach to adaptive routing," Technical report IRIDIA/97-12. IRIDIA, University Libre de Bruxelles, Brussels, 1997.

[4] S. Camazine et al., "Self-Organization in Biological Systems," Princeton University Press, 2003..

[5] I. Kassabalidis et al., "Swarm intelligence for routing in communication networks," Global Telecommunication Conference, St Antonio, 25-29 Nov., 2001, pp. 3163-3167.

[6] M. H. Roth, "Termit: a swarm intelligent routing algorithm for mobile ad-hoc networks," [Doctoral thesis], 2005, Cornell University.

[7] D. J. S. Barr, "Evolution and kingdoms of organisms from the perspective of a mycologist," Mycologia, 84, 1992, pp. 1-11.

[8] N. A. R. Gow and G. M.Gadd, "The growing fungus," Oxford: Alden Press, 1995.

[9] J. Deacon, "Fungal biology,". 4th ed. Oxford: Blackwell, 2006.

[10] D. H. Jennings. and A. D. M. Rayner, "The ecology and physiology of the fungal mycelium," 8th symposium of the British Mycological Society, Cambridge University Press, 1984

[11] R. E. Falconer et al., "Biomass recycling and the origin of phenotype in fungal mycelia," Pro. Royal Society B: Biological Sciences. 272(1537), 2005, pp. 1727-1734. 
[12] A. Adamztzky and O. Holland, O., "Reaction-diffusion and antbased load balancing of communication networks," Kybernetes. $31(5), 2002$, pp. 667-681. 